Review

\title{
Left-Right Asymmetrical Development of the Proepicardium
}

\section{Jan Schlueter and Thomas Brand *}

Heart Science Centre, National Heart and Lung Institute, Imperial College London, Hill End Road, Harefield, UB9 6JH, UK; E-Mail: j.schluter@imperial.ac.uk

* Author to whom correspondence should be addressed; E-Mail: t.brand@imperial.ac.uk;

Tel.: +44-1895-453-826; Fax: +44-1895-828-900.

Received: 30 May 2013; in revised form: 15 July 2013 / Accepted: 22 July 2013 /

Published: 26 July 2013

\begin{abstract}
The proepicardium (PE) is a cluster of cells that forms on the cardiac inflow tract and gives rise to the epicardium and connective tissue and largely contributes to the coronary vasculature. In many vertebrates, the PE undergoes left-right asymmetrical development. While PE cells and marker genes can be initially found on both sides, only the right-sided PE will fully develop and ultimately deliver cells to the heart. Several signalling inputs, like FGF and BMP signals, are involved in PE induction in the lateral plate mesoderm, as well as during inflow tract formation and, also, control asymmetric PE development. These signalling events will be put into the context of embryonic left-right asymmetry determination. Finally, it will be discussed whether PE development may serve as a readout for asymmetric inflow tract morphogenesis.
\end{abstract}

Keywords: chicken embryo; FGF8; SNAI1; PITX2; TBX18; WT1; inflow tract; sinus venosus

\section{Introduction}

Left-right (L-R) asymmetry is a basic feature of the vertebrate body plan. It encompasses asymmetric organ placement within the coelom, as well as intrinsic chirality of organs, like the heart or the gut tube [1,2]. Any failure of establishing proper organ position and asymmetric morphogenesis along the L-R axis during embryonic development may result in heterotaxia, which means 
non-concordant allocation of organ anlagen to the left and right body side. Such a condition, which is also named situs ambiguus, causes a wide spectrum of congenital heart defects, such as atrial septal defects (ASDs), ventricular septal defects (VSDs), atrioventricular septum defects (AVSD), transposition of the great arteries (TGA), double outlet right ventricle (DORV) and aortic arch (AA) anomalies [3,4]. Moreover, cardiac conduction disease is also common in heterotaxy syndrome [5].

While L-R asymmetry is present in many organisms, it remains a debated issue when and, in particular, how L-R asymmetry is established [6]. There is agreement in the field that there are three phases involved in L-R axis development [7]. An early phase exists, during which the L-R-axis is oriented relative to the anteroposterior and dorsoventral axes. The second phase involves translation of this first decision into asymmetric gene expression. In the third step, L-R asymmetry information is transduced into asymmetric morphogenesis.

Surprisingly, the first step in L-R axis determination appears to be the least conserved. Several mechanisms have been found in organisms, such as a asymmetric imprinting of chromatids, chiral cytoskeleton, asymmetric distribution of ion channels and pumps, leading to asymmetric distribution of membrane voltage, asymmetric transport of molecules, such as serotonin through gap junctions, planar cell polarity, asymmetric cell migration and, last, but not least, ciliary flow [6]. These different mechanisms of establishing the L-R axis are utilized in organisms to a different extent. In higher vertebrates, symmetry breaking occurs during early gastrulation, although the exact timing has not been fully established, due to the difficulties in studying the mammalian embryo before implantation. In mice, it is believed that the nodal flow, a directional fluid flow generated by cilia, which are precisely positioned on the ventroposterior side of nodal cells in the gastrulating mouse embryo, establishes the L-R axis [1]. An alternative mechanism present in the chicken and possibly in the porcine embryo induces asymmetric cell migration around Hensen's node, which precedes asymmetric gene expression [8]. In the chicken, asymmetric distribution of membrane voltage, asymmetric transport of serotonin through gap junctions and the planar cell polarity pathway are upstream of establishing L-R asymmetry at the node [2].

The most prominent signalling cascade involved in determining L-R asymmetry is the evolutionary conserved NODAL/PITX2 pathway, which is induced at the embryonic organizer and determines the left-sided identity of the lateral plate mesoderm (Figure 1) [9,10]. On the right side, PITX2 is actively repressed by FGF8 and $S N A 1$ in the chick embryo [11,12]. It has been shown in various animal models, including chicken and Xenopus, that these signalling cascades are preceded by asymmetric ion flux across the embryo, which may lead to an asymmetric accumulation of low molecular weight determinants, like serotonin [13].

There is only a limited amount of data on the actual impact of these L-R signalling events on asymmetric organogenesis at later developmental stages. It has been shown, for example, that the asymmetric bending of the gut tube is under the control of PITX2, which induces left-sided tissue condensation in the dorsal mesentery [14]. Gut looping in the zebrafish depends on asymmetric migration of lateral plate mesoderm, and NODAL signalling is essential for proper gut looping [15]. The molecular mechanisms that govern cardiac looping are poorly understood. Some progress has been made in the zebrafish embryo, which apparently uses NODAL and BMP signals to control asymmetric cell migration [16-18]. However, whether these pathways are evolutionary conserved and also utilized in higher vertebrates is presently unclear. 
Figure 1. Left-right asymmetric signalling events that are involved in proepicardium (PE) formation in the chick. During gastrulation and the onset of neurulation from $\mathrm{HH}$ stage 5 to 9, the NODAL/PITX2 pathway is induced by asymmetric SHH signalling at Hensen's node (HN). SHH induces NODAL at the left side of the node. NODAL induces itself in the left lateral plate mesoderm, where it induces PITX2. In order to prevent Nodal from spreading to the right side, NODAL induces a feedback inhibitor, LEFTY2, in the developing notochord (NT). On the right side of the node, FGF8 antagonizes $S H H$ and induces SNAII in the right lateral plate mesoderm. SNAI1 acts as a transcriptional repressor of PITX2 on the right side. At HH stage 11, the embryonic heart is depicted. On the left side of the embryo, including the left inflow tract, PITX2 is expressed. In the right lateral plate mesoderm, $S N A I 1$ still lingers and is subsequently downregulated. At this stage, the first PE marker gene expression can be detected. TBX18 is initially expressed on the right side, and the right-sided expression of WT1 follows shortly thereafter [23]. It has been shown that manipulation of FGF8/SNAI1 interferes with asymmetric expression of TBX18 and WT1 [23]. The mechanism of how SNAI1 might regulate asymmetric PE marker gene expression remains unclear; however, it has been suggested that SNAI1 might be responsible for asymmetric mobilization of PE progenitor cells in the lateral plate. At later stages, up to $\mathrm{HH}$ stage 17, BMP and FGF signalling have been shown to be responsible for the specification and the asymmetric PE outgrowth. BMP is required at low concentration to maintain PE marker gene expression. This is realized by a differential expression of $B M P 4$ at low levels in the PE and of $B M P 2$ at high levels in the sinus venosus. A feedback loop of FGF signalling is crucial for the survival and growth of PE cells on the right side [24]. The lack of FGF on the left side leads to massive retardation of the PE primordium and upregulation of $C A S P 3$, which induces apoptosis
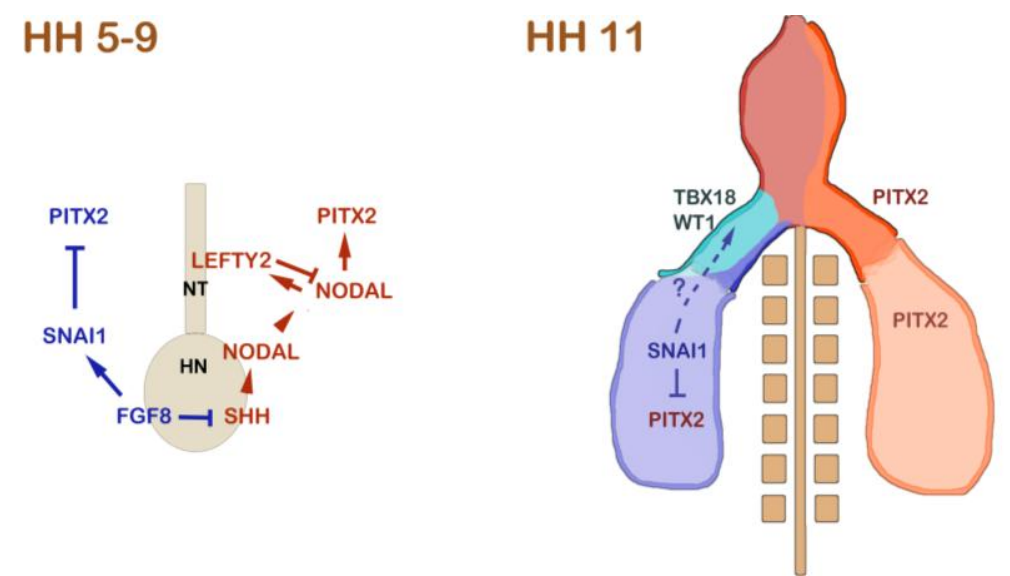

$\mathrm{HH} 17$

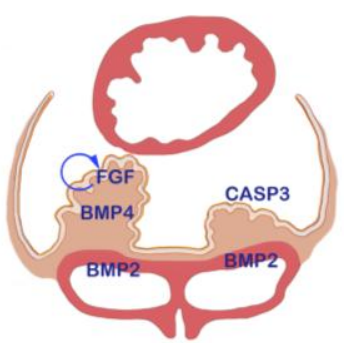

In this review, we focus on asymmetric development of the proepicardium (PE), which is an easily detectable morphological asymmetry of the embryonic heart, which is present in many vertebrate species. The PE is a cluster of progenitor cells that forms on the cardiac inflow tract. Its purpose is the accumulation and, subsequently, the transfer of progenitor cells to the embryonic heart [19]. This is accomplished via the process of epicardialization of the myocardial surface in which PE cells attach to heart and cover it with an epicardium [20]. The PE and the epicardium harbour several subpopulations of cells, which give rise to various cell types of the coronary blood vessel system, including smooth muscle 
cells, fibroblasts and endothelial cells [21] (Figure 2). The formation of the epicardium is a vital process, not only for the formation of coronary blood vessels, but also for the growth and maturation of the myocardial wall [22]. In order to ensure proper PE formation, several signalling factors, like BMP and FGF, which initially have been identified in the chick, are important for cell specification and drive proepicardial growth and survival [23-26]. In addition, PE formation in the chicken embryo has been shown to be a target of right-sided signalling during early gastrulation involving $F G F 8 / S N A I 1$, which promotes its asymmetric establishment on the cardiac inflow tract [27]. Here, we will discuss the available knowledge on asymmetric PE development among vertebrates and put it into the context of other known inflow tract asymmetries, including the formation of the cardiac pacemaker.

Figure 2. The PE is a heterogeneous cell cluster at the venous pole. The PE develops on the right sinus venosus, which is a transient region, and the connection between the vitelline veins and the atrium of the embryonic heart. At the venous pole, cells are added constantly to the heart tube. Some of these cells are also recruited into the PE. The PE consists of an outer layer of mesothelial cells, which form the characteristic protrusions that project into the pericardial cavity. The core of the PE harbours matrix-producing mesenchymal cells from the sinus venosus. Furthermore, endothelial progenitor cells are being recruited into the PE cluster, either from the sinus endothelium, the liver primordium or both. This leads to a complex mixture of vasculogenic and fibroblast progenitor cells in the PE that are ultimately transferred to the heart (modified from [23]).

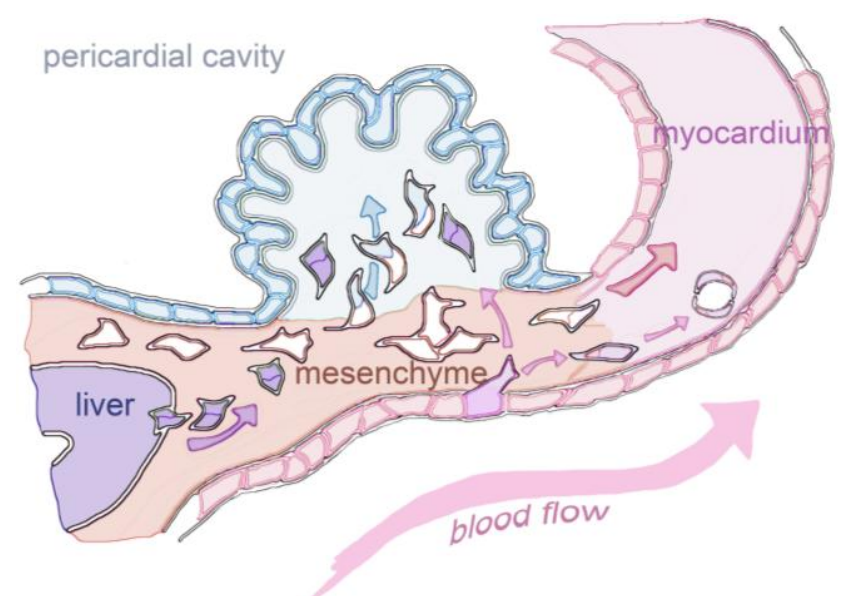

\section{Asymmetric PE Morphology among Vertebrates}

Asymmetric PE development can be observed in various vertebrate model organisms (Figure 3); however, to our knowledge, PE development has not yet been studied in the human heart. In many cases PE formation is strongly biased to the right side [28]. In the chick, the first morphological indication of PE development can be observed at the HH stage [14]. On the right side of the cardiac inflow tract, or sinus venosus, initial villous protrusions mark the morphological onset of PE formation [29]. This asymmetric morphology is preceded by the expression of conserved marker genes, like TBX18, WT1 and $T C F 21$, which start to be asymmetrically expressed in the right sinus at HH stage 12 [23].

The PE protrusions become more pronounced by enhanced proliferation, until the PE has reached its full size at $\mathrm{HH}$ stage 17, which is shortly before its attachment to the myocardium [24]. The PE at 
this stage consists of an outer layer of cuboidal mesothelial cells and an inner core of mesenchymal cells, which produces a significant amount of extracellular matrix. In addition, endothelial progenitors are present in the PE, most likely recruited from the underlying sinus of the vitelline veins, although other sources, like liver sinusoids, have also been suggested as putative site of origin [30-32]. While the right PE will subsequently attach to the dorsal side of the ventricle and colonizes the heart, the left-sided anlage displays a strongly retarded development and will not contribute any cells to the heart [21]. In the chick, the PE-derived cells are transferred to the heart via a right-sided tissue bridge that is established at HH stage 18 [21]. Since, by that stage, the PE has reached a significant size on the right inflow tract, the outer mesothelial cells eventually come in contact with the perpetually contracting ventricle. The attachment of the mesothelial villi leads to the release of matrix from the core of the PE, which facilitates the formation of the tissue bridge, along which PE cells are continuously migrating over the myocardial surface [23].

In contrast to the chick, the mouse PE does not display a prominent left-right asymmetry. The original two PE anlagen on the venous pole apparently fuse to form one medial cluster [33]. Furthermore, the transfer of cells differs from the chick. Rather than forming a tissue bridge, the highly adhesive villous protrusions are plucked off by the beating heart, resulting in the formation of a patchwork of epicardial islands, which subsequently become confluent to form a continuous epicardial layer [34]. In addition to that, the release of free-floating cell cysts from the PE, which cross the pericardial cavity and passively attach to the heart, have been described [35].

In Xenopus, like in the chick, the PE forms on the right side of the sinus venosus [36,37]. At stage 41, the PE is visible as a cone-shaped cluster of TBX18-positive mesothelial cells. The time window of PE formation is much shorter than in the chick, and the PE in Xenopus embryos can be observed only for a couple of hours at stage 41 , before it makes contact with the heart. The cellular transfer is accomplished via a tissue bridge, which persists up to stage 46. PE development in Xenopus displays the strongest bias of all animal models, since no morphological or molecular evidence for a left-sided PE anlage has been obtained. This might be due to the accelerated PE development in Xenopus, but also, it could be the result of the very early establishment of the left-right axis during the first cell division in this model organism. In fact, right-sided PE formation appears to be common among amphibians, since it can also be observed in the axolotl [38].

In various fish species, PE development appears to be less stringent in terms of bilateral asymmetry. In zebrafish, the first signs of the PE can be found after two days post-fertilization on the sinus venosus $[39,40]$. However, the spatial relation of the PE to the cardiac inflow tract and the surrounding pericardial cavity are still under investigation. Nonetheless, it has been suggested that PE cells are released into the pericardial cavity, rather than being transferred via a tissue bridge. The mechanisms by which free-floating cells or epithelial cysts are delivered to the heart remain to be investigated. The analysis of PE marker genes indicates the existence of two PE anlagen at the venous pole, but the potential contribution of both primordia to the epicardium is presently unknown [39]. The question of the bilateral origin of the PE was experimentally tested by inducing cardia bifida through knockdown of miles apart ( $m i l$ ) or casanova (cas) genes. In contrast to the chick, where in cardia bifida embryos, only the right heart develops a PE [29], in the zebrafish embryo, both heart tubes develop WT1-positive PE cells [39]. These data suggest differences between chick and zebrafish with regard to asymmetric PE development. 
Figure 3. Examples of asymmetric and symmetric PE development among vertebrates. The PE generally forms at the venous pole of the heart and is in close relation to the major veins. Asymmetric PE formation is a conserved feature of vertebrate development. Among the most prominent model organisms are the chick and Xenopus, which both exhibit a right-sided PE cluster that establishes a tissue bridge to the heart. The most basic vertebrate studied so far is the lamprey, Petromyzon marinus, which also displays a right-sided tissue bridge to the heart. Additionally, in the lamprey, the PE is in close spatial relation to the pronephros (olive), and after epicardium formation, the PE cluster gives rise to the highly vascularized pronephric glomeruli. In mouse embryos, asymmetric PE formation has not been observed; instead, the bilaterally formed PE clusters fuse at the midline of the sinus venosus, and PE cell transfer is either accomplished by the release of PE cysts into the pericardial cavity or by being plucked off by the contracting ventricle (modified from [23]).
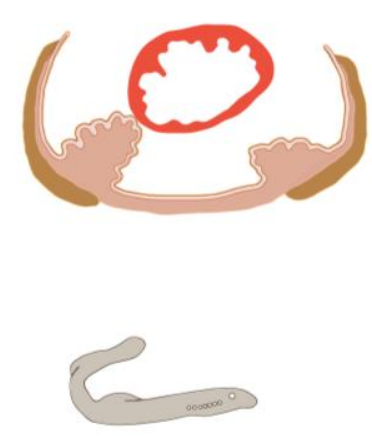

Petromyzon m.
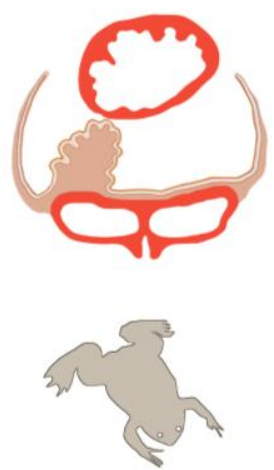

Xenopus afr.
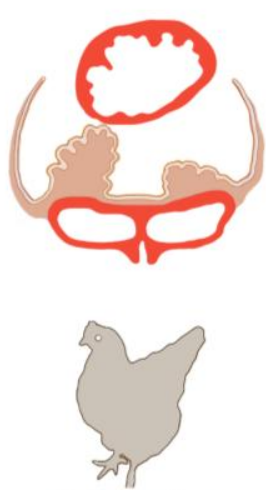

Gallus gallus
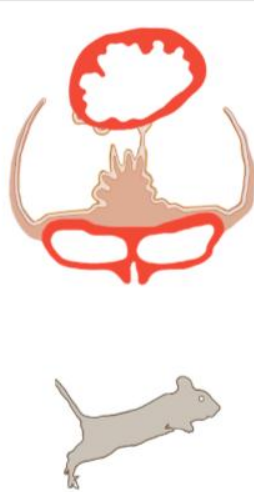

Mus musculus

The sturgeon is a more archaic fish species in which epicardium formation has been thoroughly analysed [41]. After four days post-hatching, two bilateral PE anlagens can be observed, which subsequently fuse. Although there is seemingly no evidence for an asymmetric PE induction, the sturgeon makes use of tissue bridges, which have been found to be associated with asymmetric PE development in higher vertebrates. However, tissue bridges are not the only mode of cell transfer to the heart in the sturgeon. Although the conus myocardium is covered with a continuous epicardial layer, it has been suggested that the ventricle is colonized by PE cells that have been released into the pericardial cavity. The tissue bridges are not transient, like in the chick or Xenopus, but persist as a connection between the coronary vascular plexus and the sinus venosus and, also, carry cardiac nerves [41].

From an evolutionary point of view, the lamprey, Petromyzon marinus, is the most distant and primitive of all vertebrate model organisms that have been used to study PE development [42]. The lamprey is a jawless parasitic fish, which, together with the hagfish, belongs to the group of agnathans. The lamprey has a peculiar anatomy, which brings the PE in close evolutionary relation to the pronephros. Evidence for such a relation can be found in higher vertebrates at the molecular level. For example, in the chick, the PE and the intermediate mesoderm, which gives rise to the nephric system, share the expression of several transcriptions factors, like TBX18, WT1 and TCF21 [23,43]. Moreover, the membrane protein, podoplanin, is expressed in podocytes of the kidney, as well as in the PE and the coelomic epithelium [44]. For further discussion on this subject, see the article by Cano et al. in 
this volume [45]. The lamprey initially develops two PE primordia, but similar to other organisms, only the right-sided PE cluster forms a tissue bridge and contributes to the epicardium. After epicardium formation, the PE clusters develop into the highly vascularized pronephric external glomeruli, underlining the vasculogenic potential as a conserved characteristic feature of the PE [42].

\section{The Generation of Left-Right Asymmetry}

The chick has served as one of the main models for the investigation of PE development, especially for the analysis of the molecular mechanisms underlying asymmetric PE formation. In order to clarify the molecules and factors involved in generating this biased development, we will briefly introduce the main signalling pathways that establish left-right asymmetry and, thereby, mainly focus on the chick embryo.

The core-signalling pathway in establishing left identity in the mesoderm is the highly conserved NODAL/PITX2 signalling cascade (Figure 1) [9,22]. This pathway is induced at the embryonic organizer or Hensen's node during gastrulation. Asymmetric, leftward movement of cells around the node [8] causes a displacement of cells expressing $S H H$ and $F G F 8$ and, thereby, establishing their asymmetric expression patterns on the left and right side of Hensen's node, respectively. SHH induces the TGFß molecule, NODAL, which is highly diffusible and spreads into the lateral plate mesoderm, where it induces transcription factors, like PITX2 and $B A P X 1$, which ultimately determine left identity [2].

There are several mechanisms that ensure that only one side acquires left-sided identity. The first step is the inhibition of $S H H$ on the right side of the node by FGF8, which basically inhibits the induction of the NODAL/PITX2 cascade on the right side [11]. Furthermore, FGF8 induces the transcriptional repressor, $S N A 1$, in the right lateral plate mesoderm, which represses PITX2 on the right side. Since NODAL is a diffusible morphogen, it is paramount to prevent it from spreading to the right side of the embryo. This is accomplished by NODAL itself, which induces another TGFß molecule, LEFTY2 [46]. LEFTY2 is expressed in the embryonic midline, has a higher diffusion rate than NODAL and is able to inhibit signalling by competing for the NODAL receptor complex. This biochemical activity establishes the midline barrier, which limits diffusion of NODAL towards the right side [47]. There are many more molecules and mechanisms involved in the establishment of the NODAL/PITX2 pathway on the left side of the embryo, but these cannot be discussed into further depth in the interest of brevity; the reader is referred elsewhere for further information [48].

Instead, we also want to mention earlier mechanisms that have been suggested to break initial embryonic symmetry. In chick and frog embryos, an asymmetric ion flux generated by H-/V-ATPases has been reported, which occurs before the onset of the NODAL/PITX2 expression and supposedly drives the asymmetric accumulation of low molecular weight determinants, like serotonin [13]. In addition, the presence of apoptotic cells, which reside in the primitive streak, might act as an early midline barrier (before LEFTY2 and the notochord comes into play) that prevents asymmetrically distributed small molecules from leaking to the other side [49]. However, it is still not fully understood how these events feed into the asymmetric expression of $S H H$ and $N O D A L$, although, in the chick embryo, evidence has been obtained for a control of asymmetric cell migration by asymmetric ion flux [8].

Another interesting finding is a highly conserved role for tubulin, which has been proposed to be responsible for asymmetric distribution of maternal factors during the first cell division in Xenopus [50]. 
Likewise, tubulin is involved in the chirality of Arabidopsis and asymmetric organogenesis in Caenorhabditis elegans. These observations largely contributed to the idea that asymmetric rearrangements of the cytoskeleton are the driving force of embryonic left-right asymmetry. Furthermore, it has been emphasized that the cellular mechanisms behind left-right axis determination, in fact, show resemblance with the planar cell polarity (PCP) pathway [51].

\section{Molecular Control of Asymmetric PE Development}

Since the chick has been amongst the best-studied organisms for both PE development, as well as left-right asymmetry, it has taken a leading role as a model for the investigation of right-sided PE development. The experimental induction of cardia bifida in chick embryos demonstrated that although the embryos develop two independently beating heart tubes, only the right inflow tract has a $\mathrm{PE}$, and consequently, only the right heart became covered with an epicardium [33]. Consequently, the left heart tube is devoid of an epicardium, which has a negative impact on the maturation of the myocardial wall. These experiments clearly establish that the PE has a right-sided identity and that the formation of the heart is not necessary for the induction of the PE. Nonetheless, it is tempting to propose a potential coordination of sidedness of PE formation and the direction of heart looping, which is also strictly rightward.

The initial hypothesis for the molecular control of PE development included the idea that PITX2 might act as a PE repressor on the left side, since PITX2 is strongly expressed in the left cardiac inflow tract. However, gain-of-function experiments show that the NODAL/PITX2 pathway does not interfere with PE formation [27]. Significantly, the forced expression of $S H H$ on the right side produces aberrant cardiac looping, but, at the same time, is unable to interfere with right-sided PE marker gene expression. These data provide further evidence for an independent regulation of these two events, which can be experimentally uncoupled. Even the overexpression of PITX2 in mesodermal progenitor cells does not change the asymmetric PE transcriptional program in the cardiac inflow tract [27]. The gain and loss of the right-sided $F G F 8 / S N A I 1$ pathway, however, does strongly affect right-sided PE marker gene expression. Ectopic FGF8 or SNAI1 is able to induce TBX18 and WT1 in the left sinus, and likewise, the loss of $F G F 8 / S N A I 1$ leads to a loss of these markers. These observations have led to the concept that right-sided $F G F 8$ and $S N A I 1$ are, in fact, more than repressive factors, but that they also have inductive capacity and are directly involved in asymmetric morphogenesis of the inflow tract [27]. However, the mechanisms of how these factors actually induce right-sided PE formation are still under investigation. FGF8 is expressed at gastrula stages, and SNAII is maintained in the lateral plate mesoderm until the onset of heart tube formation at stage HH10. This makes it necessary to identify potential downstream targets of SNAII that might interfere with PE development. SNAI1 is known to be involved in epithelial-mesenchymal transition (EMT) and promotion of cell invasion [52]. Therefore, one might hypothesize that SNAII is enhancing the invasion of PE progenitor cells from the lateral plate mesoderm, which would ultimately lead to an initial bias by a higher level of PE progenitor cells in the right cardiac inflow tract.

There are several other genes displaying asymmetric expression in the cardiac inflow tract, which have been shown to have an impact on PE formation. BMP4 starts to display a weak asymmetric expression in the right vitelline vein at $\mathrm{HH}$ stage 12 and can also be found in the PE at later stages [23]. 
This is particularly interesting, since $B M P 2$ shows a strong symmetric expression at the venous pole, but not in the PE. It has been demonstrated that there is a dosage-dependent function for BMP, which is crucial for the expression of PE marker genes, like TBX18 and WT1 [23]. A low dosage of BMP is necessary for the maintenance of PE markers, whereas higher concentrations drive a subset of PE cells into the myocardial lineage, which has been confirmed by in vitro experiments [23]. Notch signalling has been identified to be responsible for suppressing BMP in the PE in order to limit myocardialization of the cell cluster [53].

Another important group of factors that are asymmetrically expressed in the cardiac inflow tract are FGFs. Several FGF ligands and receptors are expressed in the PE and the right sinus venosus [24]. The most prominent are FGF2, which is expressed in the entire PE, and FGF10 and FGF12, which are enriched in the villous protrusions. The receptors, FGFR1, 2 and 4, are particularly expressed in the outer villi of the $\mathrm{PE}$, as well. In vitro experiments and embryonic manipulations confirmed that these FGFs are crucial for proliferation and survival of the PE cluster [24]. The inhibition of FGF signalling on the right side causes a drastic increase in the number of apoptotic cells in the right sinus. This leads to a smaller and misshaped PE, which basically resembles the fate of the PE anlage on the left side. The left sinus is strongly apoptotic in wild-type embryos, which can be explained by the absence or vastly reduced levels of FGF signalling. Given the expression patterns of ligands and receptors in the PE, an FGF auto-regulatory loop has been suggested. This would mean that the PE is self-stimulating its own survival on the right sinus to ensure proper villous outgrowth.

By now, a significant amount of data on asymmetric PE development has been accumulated; however, further investigations are necessary to provide a better understanding of how early left-right signalling impacts asymmetric PE formation at the venous pole of the heart.

\section{Additional Examples of Asymmetric Inflow Tract Development}

The heart is a highly asymmetric organ, right from the initial looping of the heart tube up to the formation of atrial and ventricular chambers at later developmental stages. The arterial and the venous pole of the heart are both undergoing massive asymmetric rearrangements during development. We would like to focus on some examples of asymmetric inflow tract development, which also might be relevant for PE development.

We have already discussed the hypothesis that there might be an asymmetric contribution of PE progenitor cells to the inflow tract under the control of the right-sided FGF8/SNAII pathway in the chick. It has recently been reported that there is an asymmetric contribution of cells from the posterior second heart field to both poles of the murine heart [54]. This is particularly interesting, since it has been proposed for the mouse embryo that there is a cranio-caudal patterning of the $I s l l$-expressing second heart field by $F g f 8, F g f 10, T b x l$ and $M e f 2 c$, which label the anterior part [55,56]. The most caudal portion of the second heart field, however, gives rise to the sinus venosus and, most likely, also, harbours PE progenitor cells [54]. This is also in agreement with the detection of Isl1-Cre labelled cells in the murine PE [57]. Since Isll has a rather broad expression domain in the lateral plate mesoderm, additional investigation is necessary to fully understand the potential contribution of the secondary heart field to the PE. 
Pitx2 has been identified as an important determinant of asymmetric inflow tract morphogenesis. Since Pitx2 is expressed in the left atrium and the left venous system, the loss of Pitx2 has multiple severe impacts on these tissues. These include atrial isomerism and deformation of the atrio-ventricular valve in Pitx2 mutants in mice [58]. In addition, deficiencies of pulmonary myocardium formation have been observed, likely due to a proliferative defect in the pulmonary mesenchyme [59].

Pitx2 is also responsible for the lateralization of the cardiac pacemaker, which develops at the venous pole [60]. At developmental stages before the sinus venosus becomes lateralized, the entire caudal region of the tubular heart displays pacemaker activity. Initially, the dominant pacemaker is on the left side [61]. Pacemaker activity gradually becomes confined to the sinoatrial node (SAN) on the right side, with the progressive maturation of the sinus venosus myocardium. During this process, Pitx2 is required for the establishment of left identity of the left sinus and the future left atrium, thereby limiting the pacemaking capacity to the future SAN on the right side. Pitx2 is able to bind to Shox2, a master regulator of SAN development, thereby directly inhibiting a SAN-specific transcriptional program in the left sinus. This asymmetric inhibition has been shown to be crucial to ensure proper pacing of the heart and to prevent atrial fibrillation, a highly prevalent type of cardiac arrhythmia [62].

It has recently been shown that the progenitor cells of the cardiac pacemaker in the chick are localized in a region posterior to the heart forming fields and that these cells are very early specified by canonical WNT signalling [63]. This is in close proximity to the presumptive origin of sinus venosus progenitor cells in the posterior region of the heart field [64]. It would be interesting to investigate whether the fate of sinus venosus cells that contribute to the PE is likewise under the control of WNT signals at the early gastrula stage. Although both PE and pacemaker cells are crucial constituents of the right cardiac inflow tract, their asymmetric development is controlled by different, even antagonistic signalling pathways. Ultimately, a master regulator, like PITX2, has not been identified yet for the right side. The impact of the rather early acting $F G F 8 / S N A I 1$ pathway and the lack of responsiveness to PITX2 suggest a more complex scenario for the asymmetric development of the PE than mere transcriptional repression. As already outlined, this includes asymmetric specification and survival by BMP and FGF, as well as the postulated invasion of PE progenitor cells into the right sinus. However, this is not unexpected, since the PE is a heterogeneous cell cluster and composed of cells with potentially diverse origins, which have yet to be fully defined. The PE has a very distinct transcriptional program, including factors like TBX18,WT1, TCF21 and CFC [23]. This transcriptional program is shared to some extent with the coelomic pericardial mesoderm, as well as the nephrogenic intermediate mesoderm, both tissues that lie outside the heart forming fields. Therefore, the investigation of the heterogeneity and the origin of the PE might hold the key to understand the diverse differentiation potential of this cell cluster and underline its significance as a highly conserved extracardiac cell population accumulating at the cardiac inflow tract.

L-R asymmetry of the PE is found in a number vertebrate species. However, until now, the underlying molecular pathway has only been investigated in the chick embryo. An important question in the future will be whether the pathway of asymmetric PE development is conserved in other species. In this regard the Xenopus embryo will be a very valuable model organism for such a comparative analysis. In this species, the L-R axis has already been laid down together with the first cell division, and PE development is asymmetric, as in the chick embryo. Will SNAII in Xenopus be upstream of PE 
development? Is PE development in this species independent of Pitx2, as has been shown in the chick embryo? Likewise, the mouse needs to be studied further. SNAI1 is also in the mouse embryo upstream of L-R asymmetry [59]. In the SNAI1 knockout mutant, both heart looping and embryo turning are severely abnormal. PE development in the mouse is clearly symmetric [29]; however, this might be a secondarily acquired pattern. There is the possibility of an underlying asymmetry, which secondarily is suppressed by some other mechanism. Such a phenomenon is, for example, seen in the case of somite development, which is inherently asymmetric, but asymmetry is suppressed by retinoic acid [60,61]. Another important area for future research is to identify the mechanism through which SNAII has an impact on PE development. It is possible that $S N A I 1$ will induce some other transcription factor, since expression is not maintained until PE-specific gene expression commences. Another possibility is, of course, that downstream of SNAI1 is a paracrine factor, such as BMP4 [19]. Single cell resolution can probably only be accomplished in the zebrafish, and therefore, it will be important for defining proepicardial cell lineages to generate transgenic GFP lines in the zebrafish that specifically label the PE [62,63]. Such lines will ultimately be needed to define the origin of different PE populations and how asymmetric or symmetric PE formation is accomplished.

\section{Acknowledgments}

This work was supported by Imperial College London and the Magdi Yacoub Foundation.

\section{Conflict of Interest}

The authors declare no conflict of interest.

\section{References and Notes}

1. Nakamura, T.; Hamada, H. Left-right patterning: conserved and divergent mechanisms. Development 2012, 139, 3257-3262.

2. Schlueter, J.; Brand, T. Left-right axis development: Examples of similar and divergent strategies to generate asymmetric morphogenesis in chick and mouse embryos. Cytogenet. Genome Res. 2007, 117, 256-267.

3. Gelb, B.D. Molecular genetics of congenital heart disease. Curr. Opin. Cardiol 1997, 12, 321-328.

4. Ramsdell, A.F. Left-right asymmetry and congenital cardiac defects: Getting to the heart of the matter in vertebrate left-right axis determination. Dev. Biol. 2005, 288, 1-20.

5. Kurosawa, H.; Kawada, N. The conduction system in heterotaxy. World J. Pediatr. Congenit. Heart Surg. 2011, 2, 275-277.

6. Vandenberg, L.N.; Levin, M. A unified model for left-right asymmetry? Comparison and synthesis of molecular models of embryonic laterality. Dev. Biol. 2013, 379, 1-15.

7. Levin, M.; Palmer, A.R. Left-right patterning from the inside out: Widespread evidence for intracellular control. Bioessays 2007, 29, 271-287.

8. Gros, J.; Feistel, K.; Viebahn, C.; Blum, M.; Tabin, C.J. Cell movements at Hensen's node establish left/right asymmetric gene expression in the chick. Science 2009, 324, 941-944. 
9. Gritsman, K.; Talbot, W.S.; Schier, A.F. Nodal signaling patterns the organizer. Development 2000, 127, 921-932.

10. Logan, M.; Pagan-Westphal, S.M.; Smith, D.M.; Paganessi, L.; Tabin, C.J. The transcription factor Pitx2 mediates situs-specific morphogenesis in response to left-right asymmetric signals. Cell 1998, 94, 307-317.

11. Boettger, T.; Wittler, L.; Kessel, M. FGF8 functions in the specification of the right body side of the chick. Curr. Biol. 1999, 9, 277-280.

12. Patel, K.; Isaac, A.; Cooke, J. Nodal signalling and the roles of the transcription factors SnR and Pitx2 in vertebrate left-right asymmetry. Curr. Biol. 1999, 9, 609-612.

13. Fukumoto, T.; Kema, I.P.; Levin, M. Serotonin signaling is a very early step in patterning of the left-right axis in chick and frog embryos. Curr. Biol. 2005, 15, 794-803.

14. Davis, N.M.; Kurpios, N.A.; Sun, X.; Gros, J.; Martin, J.F.; Tabin, C.J. The chirality of gut rotation derives from left-right asymmetric changes in the architecture of the dorsal mesentery. Dev. Cell. 2008, 15, 134-145.

15. Horne-Badovinac, S.; Rebagliati, M.; Stainier, D.Y. A cellular framework for gut-looping morphogenesis in zebrafish. Science 2003, 302, 662-665.

16. Bakkers, J.; Verhoeven, M.C.; Abdelilah-Seyfried, S. Shaping the zebrafish heart: From left-right axis specification to epithelial tissue morphogenesis. Dev. Biol. 2009, 330, 213-220.

17. Veerkamp, J.; Rudolph, F.; Cseresnyes, Z.; Priller, F.; Otten, C.; Renz, M.; Schaefer, L.; Abdelilah-Seyfried, S. Unilateral dampening of Bmp activity by nodal generates cardiac left-right asymmetry. Dev. Cell. 2013, 24, 660-667.

18. Lenhart, K.F.; Holtzman, N.G.; Williams, J.R.; Burdine, R.D. Integration of nodal and BMP signals in the heart requires FoxH1 to create left-right differences in cell migration rates that direct cardiac asymmetry. PLoS Genet. 2013, 9, e1003109.

19. Mikawa, T.; Gourdie, R.G. Pericardial mesoderm generates a population of coronary smooth muscle cells migrating into the heart along with ingrowth of the epicardial organ. Dev. Biol. 1996, 174, 221-232.

20. Nahirney, P.C.; Mikawa, T.; Fischman, D.A. Evidence for an extracellular matrix bridge guiding proepicardial cell migration to the myocardium of chick embryos. Dev. Dyn. 2003, 227, 511-523.

21. Männer, J. Does the subepicardial mesenchyme contribute myocardioblasts to the myocardium of the chick embryo heart? A quail-chick chimera study tracing the fate of the epicardial primordium. Anat. Rec. 1999, 255, 212-226.

22. Manner, J.; Schlueter, J.; Brand, T. Experimental analyses of the function of the proepicardium using a new microsurgical procedure to induce loss-of-proepicardial-function in chick embryos. Dev. Dyn. 2005, 233, 1454-1463.

23. Schlueter, J.; Manner, J.; Brand, T. BMP is an important regulator of proepicardial identity in the chick embryo. Dev. Biol. 2006, 295, 546-558.

24. Torlopp, A.; Schlueter, J.; Brand, T. Role of fibroblast growth factor signaling during proepicardium formation in the chick embryo. Dev. Dyn. 2010, 239, 2393-2403.

25. van Wijk, B.; van den Berg, G.; Abu-Issa, R.; Barnett, P.; van der Velden, S.; Schmidt, M.; Ruijter, J.M.; Kirby, M.L.; Moorman, A.F.; van den Hoff, M.J. Epicardium and myocardium 
separate from a common precursor pool by crosstalk between bone morphogenetic protein-and fibroblast growth factor-signaling pathways. Circ. Res. 2009, 105, 431-441.

26. Kruithof, B.P.; van Wijk, B.; Somi, S.; Kruithof-de Julio, M.; Pérez Pomares, J.M.; Weesie, F.; Wessels, A.; Moorman, A.F.; van den Hoff, M.J.; BMP and FGF regulate the differentiation of multipotential pericardial mesoderm into the myocardial or epicardial lineage. Dev. Biol. 2006, 295, 507-522.

27. Schlueter, J.; Brand, T. A right-sided pathway involving FGF8/Snail controls asymmetric development of the proepicardium in the chick embryo. Proc. Natl. Acad. Sci. USA 2009, 106, 7485-7490.

28. Schlueter, J.; Brand, T. Epicardial progenitor cells in cardiac development and regeneration. $J$. Cardiovasc. Transl. Res. 2012, 5, 641-653.

29. Männer, J.; Perez-Pomares, J.M.; Macias, D.; Munoz-Chapuli, R. The origin, formation and developmental significance of the epicardium: a review. Cells Tissues Organs 2001,169, 89-103.

30. Katz, T.C.; Singh, M.K.; Degenhardt, K.; Rivera-Feliciano, J.; Johnson, R.L.; Epstein, J.A.; Tabin, C.J. Distinct compartments of the proepicardial organ give rise to coronary vascular endothelial cells. Dev. Cell. 2012, 22, 639-650.

31. Cossette, S.; Misra, R. The identification of different endothelial cell populations within the mouse proepicardium. Dev. Dyn. 2011, 240, 2344-2353.

32. Perez-Pomares, J.M.; Carmona, R.; Gonzalez-Iriarte, M.; Macias, D.; Guadix, J.A.; Munoz-Chapuli, R. Contribution of mesothelium-derived cells to liver sinusoids in avian embryos. Dev. Dyn. 2004, 229, 465-474.

33. Schulte, I.; Schlueter, J.; Abu-Issa, R.; Brand, T.; Manner, J. Morphological and molecular leftright asymmetries in the development of the proepicardium: A comparative analysis on mouse and chick embryos. Dev. Dyn. 2007, 236, 684-695.

34. Rodgers, L.S.; Lalani, S.; Runyan, R.B.; Camenisch, T.D. Differential growth and multicellular villi direct proepicardial translocation to the developing mouse heart. Dev. Dyn. 2008, 237, 145-152.

35. Hirose, T.; Karasawa, M.; Sugitani, Y.; Fujisawa, M.; Akimoto, K.; Ohno, S.; Noda, T. PAR3 is essential for cyst-mediated epicardial development by establishing apical cortical domains. Development 2006, 133, 1389-1398.

36. Jahr, M.; Schlueter, J.; Brand, T.; Manner J. Development of the proepicardium in Xenopus laevis. Dev. Dyn. 2008, 237, 3088-3096.

37. Tandon, P.; Miteva, Y.V.; Kuchenbrod, L.M.; Cristea, I.M.; Conlon, F.L. Tcf21 regulates the specification and maturation of proepicardial cells. Development 2013, 140, 2409-2421.

38. Fransen, M.E.; Lemanski, L.F. Epicardial development in the axolotl, Ambystoma mexicanum. Anat. Rec. 1990, 226, 228-236.

39. Serluca, F.C. Development of the proepicardial organ in the zebrafish. Dev. Biol. 2008, 315, 18-27.

40. Liu, J.; Stainier, D.Y. Tbx5 and Bmp signaling are essential for proepicardium specification in zebrafish. Circ. Res. 2010, 106, 1818-1828.

41. Icardo, J.M.; Guerrero, A.; Duran, A.C.; Colvee, E.; Domezain, A.; Sans-Coma, V. The development of the epicardium in the sturgeon Acipenser naccarii. Anat. Rec. 2009, 292, 1593-1601. 
42. Pombal, M.A.; Carmona, R.; Megias, M.; Ruiz, A.; Perez-Pomares, J.M.; Munoz-Chapuli, R. Epicardial development in lamprey supports an evolutionary origin of the vertebrate epicardium from an ancestral pronephric external glomerulus. Evol. Dev. 2008, 10, 210-216.

43. Robb, L.; Mifsud, L.; Hartley, L.; Biben, C.; Copeland, N.G.; Gilbert, DJ.; Jenkins, N.A.; Harvey, R.P. Epicardin: A novel basic helix-loop-helix transcription factor gene expressed in epicardium, branchial arch myoblasts, and mesenchyme of developing lung, gut, kidney, and gonads. Dev. Dyn. 1998, 213, 105-113.

44. Mahtab, E.A.; Wijffels, M.C.; Van Den Akker, N.M.; Hahurij, N.D.; Lie-Venema, H.; Wisse, L.J.; De Ruiter, M.C.; Uhrin, P.; Zaujec, J.; Binder, B.R.; Schalij, M.J.; Poelmann, R.E.; Gittenberger-De Groot, A.C. Cardiac malformations and myocardial abnormalities in podoplanin knockout mouse embryos: Correlation with abnormal epicardial development. Dev. Dyn. 2008, 237, 847-857.

45. Cano, E.; Carmona, R.; Munoz-Chamuli, R. Evolutionary origin of the pronephros. J. Dev. Biol. 2013, 1, 3-19.

46. Schlange, T.; Schnipkoweit, I.; Andree, B.; Ebert, A.; Zile, M.H.; Arnold, H.H.; Brand, T. Chick CFC controls Leftyl expression in the embryonic midline and nodal expression in the lateral plate. Dev. Biol. 2001, 234, 376-389.

47. Muller, P.; Rogers, K.W.; Jordan, B.M.; Lee, J.S.; Robson, D.; Ramanathan, S.; Schier, A.F. Differential diffusivity of Nodal and Lefty underlies a reaction-diffusion patterning system. Science 2012, 336, 721-724.

48. Okada, Y.; Takeda, S.; Tanaka, Y.; Izpisua Belmonte, J.C.; Hirokawa, N. Mechanism of nodal flow: A conserved symmetry breaking event in left-right axis determination. Cell 2005, 121, 633-644.

49. Kelly, K.A.; Wei, Y.; Mikawa, T. Cell death along the embryo midline regulates left-right sidedness. Dev. Dyn. 2002, 224, 238-244.

50. Lobikin, M.; Wang, G.; Xu, J.; Hsieh, Y.W.; Chuang, C.F.; Lemire, J.M.; Levin, M. Early, nonciliary role for microtubule proteins in left-right patterning is conserved across kingdoms. Proc. Natl. Acad. Sci. USA 2012, 109, 12586-12591.

51. Aw, S.; Levin, M. Is left-right asymmetry a form of planar cell polarity? Development 2009, 136, $355-366$.

52. Dave, N.; Guaita-Esteruelas, S.; Gutarra, S.; Frias, À.; Beltran, M.; Peiró, S.; de Herreros, A.G. Functional cooperation between Snaill and twist in the regulation of ZEB1 expression during epithelial to mesenchymal transition. J. Biol. Chem. 2011, 286, 12024-12032.

53. del Monte, G.; Casanova, J.C.; Guadix, J.A.; MacGrogan, D.; Burch, J.B.; Pérez-Pomares, J.M.; de la Pompa, J.L. Differential Notch signaling in the epicardium is required for cardiac inflow development and coronary vessel morphogenesis. Circ. Res. 2011, 108, 824-836.

54. Dominguez, J.N.; Meilhac, S.M.; Bland, Y.S.; Buckingham, M.E.; Brown, N.A. Asymmetric fate of the posterior part of the second heart field results in unexpected left/right contributions to both poles of the heart. Circ. Res. 2012, 111, 1323-1335.

55. Watanabe, Y.; Miyagawa-Tomita, S.; Vincent, S.D.; Kelly, R.G.; Moon, A.M.; Buckingham, M.E. Role of mesodermal FGF8 and FGF10 overlaps in the development of the arterial pole of the heart and pharyngeal arch arteries. Circ. Res. 2010, 106, 495-503. 
56. Lazic, S.; Scott, I.C. Mef2cb regulates late myocardial cell addition from a second heart field-like population of progenitors in zebrafish. Dev. Biol. 2011, 354, 123-133.

57. Zhou, B.; von Gise, A.; Ma, Q.; Rivera-Feliciano, J.; Pu, WT. Nkx2-5- and Isl1-expressing cardiac progenitors contribute to proepicardium. Biochem. Biophys Res. Commun. 2008, 375, $450-453$.

58. Campione, M.; Ros, M.A.; Icardo, J.M.; Piedra, E.; Christoffels, V.M.; Schweickert, A.; Blum, M.; Franco, D.; Moorman, A.F. Pitx2 expression defines a left cardiac lineage of cells: Evidence for atrial and ventricular molecular isomerism in the iv/iv mice. Dev. Biol. 2001, 231, 252-264.

59. Mommersteeg, M.T.; Brown, N.A.; Prall, O.W.; de Gier-de Vries, C.; Wiese, C.; Clout, D.E.; Papaioannou, V.E.; Brown, N.A.; Harvey, R.; Moorman, A.F.; Christoffels, V.M. Pitx2c and Nkx2-5 are required for the formation and identity of the pulmonary myocardium. Circ. Res. 2007, 101, 902-909.

60. Mommersteeg, M.T.; Hoogaars, W.M.; Prall, O.W.; de Gier-de Vries, C.; Wiese, C.; Clout, D.E.; Papaioannou, V.E.; Brown, N.A.; Harvey, R.P.; Moorman, A.F.; Christoffels, V.M. Molecular pathway for the localized formation of the sinoatrial node. Circ. Res. 2007, 100, 354-362.

61. Kamino, K.; Hirota, A.; Fujii, S. Localization of pacemaking activity in early embryonic heart monitored using voltage-sensitive dye. Nature 1981, 290, 595-597.

62. Wang, J.; Klysik, E.; Sood, S.; Johnson, R.L.; Wehrens, X.H.; Martin, J.F. Pitx2 prevents susceptibility to atrial arrhythmias by inhibiting left-sided pacemaker specification. Proc. Natl. Acad. Sci. USA 2010, 107, 9753-9758.

63. Bressan, M.; Liu, G.; Mikawa, T. Early mesodermal cues assign avian cardiac pacemaker fate potential in a tertiary heart field. Science 2013, 340, 744-748.

64. Redkar, A.; Montgomery, M.; Litvin, J. Fate map of early avian cardiac progenitor cells. Development 2001, 128, 2269-2279.

(C) 2013 by the authors; licensee MDPI, Basel, Switzerland. This article is an open access article distributed under the terms and conditions of the Creative Commons Attribution license (http://creativecommons.org/licenses/by/3.0/). 